\title{
In silico studies and biological evaluation of chalcone-based 1,5-benzothiazepines as new potential H1N1 neuraminidase inhibitors
}

\author{
Neni Frimayanti ${ }^{1}$, Marzieh Yaeghoobi ${ }^{2 *}$, Hamid Namavar ${ }^{2}$, Ihsan Ikhtiarudin ${ }^{1}$, Meysam Afzali ${ }^{2}$ \\ ${ }^{1}$ Sekolah Tinggi Ilmu Farmasi Riau, Riau, Indonesia. \\ ${ }^{2}$ Faculty of Medicine, Shahroud University of Medical Science, Shahroud, Iran.
}

\begin{tabular}{l}
\hline ARTICLE INFO \\
\hline Received on: 06/05/2020 \\
Accepted on: $11 / 07 / 2020$ \\
Available online: 05/10/2020
\end{tabular}

Key words:

H1N1, neuraminidase, docking, Molecular dynamic, ADME.

\begin{abstract}
1,5-Benzothiazepines are benzo-fused seven-membered heterocycles. Due to their biological activities against a wide spectrum of targets, they are of particular interest for drug discovery. However, their antiviral activities and in silico studies of the binding to their biological targets have not been extensively investigated. In this study, we tested antiviral activities of twelve 1,5 benzothiazepine compounds (1,5-benzothiazepines) against the H1N1 influenza virus also known as swine flu. The target of chalcone-based 1,5-benzothiazepines was viral protein, H1N1 neuraminidase, and the binding was monitored using 4-methylumbelliferyl)-a-D-N-acetylneuraminic acid assay [2'-(4-methylumbelliferyl)$\alpha$-D-N-acetylneuraminic acid]. 2,3-didehydro-2-deoxy-N-acetylneuraminic acid (DANA) has been used as a positive control. Before the bioassay, computational pipelines were drawn to identify the potential bioactive compounds. Results from the docking revealed that compound MA10 bound favorably to the active site on H1N1 neuraminidase and the free energy of binding was $-8.8 \mathrm{kcal} / \mathrm{mol}$. We have also shown the importance of hydrophobic interactions, hydrogen bonds, and aromatic ring features for enhancing the biological activity of the pharmocophore. MA10 was also stable in the molecular dynamics simulation with the lowest binding energy conformation (i.e., energy minimum to bound conformation with the protein). Moreover, based on the Lipinski's rule of five, MA10 showed druglikeness properties. Although neither of the tested benzothiazepines reached the inhibitory activity measured for DANA, their in silico behavior accorded well with that in in vitro assays. Among all the compounds tested with H1N1 neuraminidase, MA10 exhibited the best inhibitory activity, so this benzolog could be used against H1N1. We, therefore, suggest an in vitro plus in silico strategy to be employed in the early-stage drug-discovery process, which might help us to identify drug candidates successfully with minimum research time and costs.
\end{abstract}

\section{INTRODUCTION}

The H1N1 virus spreads very quickly among humans through coughing or sneezing and may cause acute respiratory infections with serious health complications. H1N1 is a type of influenza A virus widely known as swine flu, which is pathogenic not only for humans but also for poultry and mammals such as horses and pigs (Maksum, 2010). The first pandemic of H1N1 dates back to 1918 (Taubenberger et al., 2006), and since then, many new strains of the virus emerged, which can be transmitted from human to human (Peter et al., 2009).

\section{"Corresponding Author}

Marzieh Yaeghoobi, Faculty of Medicine, Shahroud University of Medical Science, Shahroud, Iran. E-mail: m.yaeghoobi@gmail.com
The H1N1 virus expresses two key proteins that are vital to its survival. The first is hemagglutinin, which is responsible for the virus binding to the surface of the host cell (recognition phase). The sialic acid ( $\mathrm{Sia}$ ) residues are very often found on termini of the host cell surface glycoconjugates (glycoproteins and glycolipids), and these are the sites of hemagglutininin attachment to the host cells. Another key viral protein that enables it to spread inside the human body is neuraminidase. After the replication, in order to escape the host cells, the virus needs to remove terminal Sia from host glycoconjugates, which is done by neuraminidase (Leotis et al., 2017). Hence, the neuraminidase became a target for the treatment of H1N1 influenza, and neuraminidase inhibitors such as oseltamivir and zanamivir were the first successful drugs against H1N1 (Yu et al., 2009). Unfortunately, oseltamivir/zanamivirresistant strains of $\mathrm{H} 1 \mathrm{~N} 1$ are emerging, so the evaluation of potential new neuraminidase inhibitors is of high priority. 
1,5-Benzothiazepines belong to one of the most versatile classes of organic compounds. They were broadly used as antifeedant (Reddy et al., 1993), tranquilizers (Kugita et al., 1971), antidepressants (Geyer et al., 1970), stimulants in the central nervous system (Kawashima. Co. Lt., 1985), calcium channel blockers (Kugita et al., 1971), and antimicrobial agents (Dandia et al., 1998). Many small hydrophobic/lipophilic compounds display analgesic and sedative actions because they can penetrate into the brain cells by dissolving in the phospholipid bilayers of cell membranes (Fernández and Ruiz, 2006; Page et al., 2002), and the benzothiazepines satisfy these criteria. Besides, they have been reported to combine with other bioactive compounds to form adducts, which may have enhanced pharmaceutical properties (Cherkupally et al., 2008). Chalcones are biologically valuable precasure and comprise two benzene rings (A and B) which linked through an $\alpha, \beta$-unsaturated carbonyl group (Chintakrindi et al., 2018).

Currently, the in silico studies have become an initial and crucial part of the drug discovery, and they affect the entire process of drug design and development (Wermuth, 1998). This is mostly since in silico offers significant cost, effort, and time reductions and a more green alternative compared to synthetic chemistry. Finally, the most important consideration with in silico approach is that it spares experimental animals (Neni et al., 2020).

Recently, several studies have been performed to discover new agents against H1N1 neuraminidase, but chalcone-based 1,5-benzothiazepines have not been extensively investigated. Furthermore, there is no report on in silico studies of this class of benzologs as $\mathrm{H} 1 \mathrm{~N} 1$ neuraminidase inhibitors. In the study presented herein, we aimed to test the potency of twelve chalcone-based 1,5-benzothiazepines in an inhibition of H1N1 neuraminidase through in vitro 4-methylumbelliferyl)a-D-N-acetylneuraminic acid (MUNANA) assay. The in silico studies were also applied here to study the interactions of these compounds with the viral neuraminidase.

\section{MATERIALS AND METHODS}

All twelve 1,5-benzothiazepines were synthesized, purified, and characterized following the reported procedure (Yaeghoobi et al., 2012). Their structures are shown in Table 1.

\section{Molecular docking}

Thedocking oftheligandstructures(1,5-benzothiazepines) onto the active site (catalytic triad) of H1N1 neuraminidase was achieved using molecular operating environment 2019.0101 software package (Chemical Computing Group). The docking process was initiated with the preparation of the protein and ligand. The neuraminidase structure was downloaded from the Protein Data Bank (PDB) (https://www.rcsb.org/structure/3B7E), ID $3 \mathrm{~B} 7 \mathrm{E}$, and then, we added the missing hydrogen atoms. Before the docking, we have also minimized the hydrogen bonds and fixed the alpha carbons and the backbone atoms.

Twelve 1,5-benzothiazepine compounds were docked as ligands. Their molecular structures were sketched and minimized using ChemBioDraw (Table 1). To set up the docking parameters, we employed Chemistry at HARvard Macromolecular Mechanics (CHARMM27) as the best force field. We set up a grid box dimensions enclosed with the active site of the enzyme to be $26.85 \AA$, $28.17 \AA$, and $24.53 \AA$ along the $\mathrm{x}, \mathrm{y}$, and $\mathrm{z}$ axes, respectively. From all of the conformations that emerged from the docking results, we have selected the one with the lowest binding free energy. Then, the respective enzyme-ligand adduct was minimized to a gradient of $0.01 \mathrm{kcal} / \mathrm{mol} / \AA$ using the same force field.

\section{Molecular dynamic simulation}

Preliminary molecular dynamic simulations were performed using Nanoscale Molecular Dynamics program v 2.9 with CHARMM27 used as the force field. The modeled protein was solvated in a box of TIP3P water molecules that extended more than $2.5 \AA$ away from the coordinated structure in each direction.

The modeled systems (neuraminidase-benzolog adducts) were subjected to a simulated gradual heating in the constant temperature, constant volume (number volume temperature) ensembling from 0 to $300 \mathrm{~K}$ over 100 ps. Finally, 50-ns time scale molecular dynamic (MD) simulations were carried out for each system in an isothermal isobaric ensemble (number pressure temperature) with periodic boundary conditions. The temperature and pressure coupling parameters were set at $1.0 \mathrm{ps}$. The coordinates were saved at every 0.1 ps during the sampling process. The simulations generated the conformations, which then were used for the further calculations of free energy of binding and decomposition.

\section{Pharmacophore generation}

Pharmacophore query editor tool was utilized to generate the pharmacophore properties of the chalcone-based 1,5-benzothiazepines. It was used to carry out the features of the pharmacophore and also to conduct the hypothesis for the pharmacophore alignments. In this study, the pharmacophore alignments were achieved using three features, i.e., hydrophobic atoms, hydrogen bond donors, and aromatic rings (Neni et al., 2020).

\section{Absorption, distribution, metabolism, and excretion (ADME) prediction}

ADME profiles were used to predict the physicochemical and pharmacokinetic properties of the drug candidates, i.e., chalcone-based 1,5-benzothiazepines and also to predict their druglikeness. We performed the ADME predictions using the SwissADME server (http://www.swissadme.ch/index.php).

\section{H1N1 neuraminidase assay}

MUNANA assay has been used to evaluate the potency of the chalcone-based 1,5-benzothiazepines to inhibit the activity of H1N1 neuraminidase. We tested DANA in parallel as it is a wellknown (standard) inhibitor of neuraminidase. The stock solutions of the compounds $(1 \mathrm{mg} / \mathrm{ml})$ were made in $2.5 \%$ dimethyl sulfoxide and then were diluted in the appropriate buffer before being used in the assay as samples. About $25 \mu 1$ of the sample solution was mixed with $25 \mu \mathrm{l}$ of neraminidase from H1N1 (sinobiological/ biological solution specialist) in the presence of buffer solution in a 96-microplate well. About $50 \mu \mathrm{l}$ of the substrate solution in $32.5 \mathrm{mM}$ 2-(N-morpholino)ethanesulfonic acid buffer ( $\mathrm{pH}$ 6.5) (Sigma, M8639) was then added to each well. The substrate was 2'-(4-methylumbelliferyl)- $\alpha$-D-N-acetylneuraminic acid (MUNANA, Sigma, M8639). The plate was incubated at $37^{\circ} \mathrm{C}$ for 1 
Table 1. Molecular structures of chalcone-based 1,5-benzothiazepines.

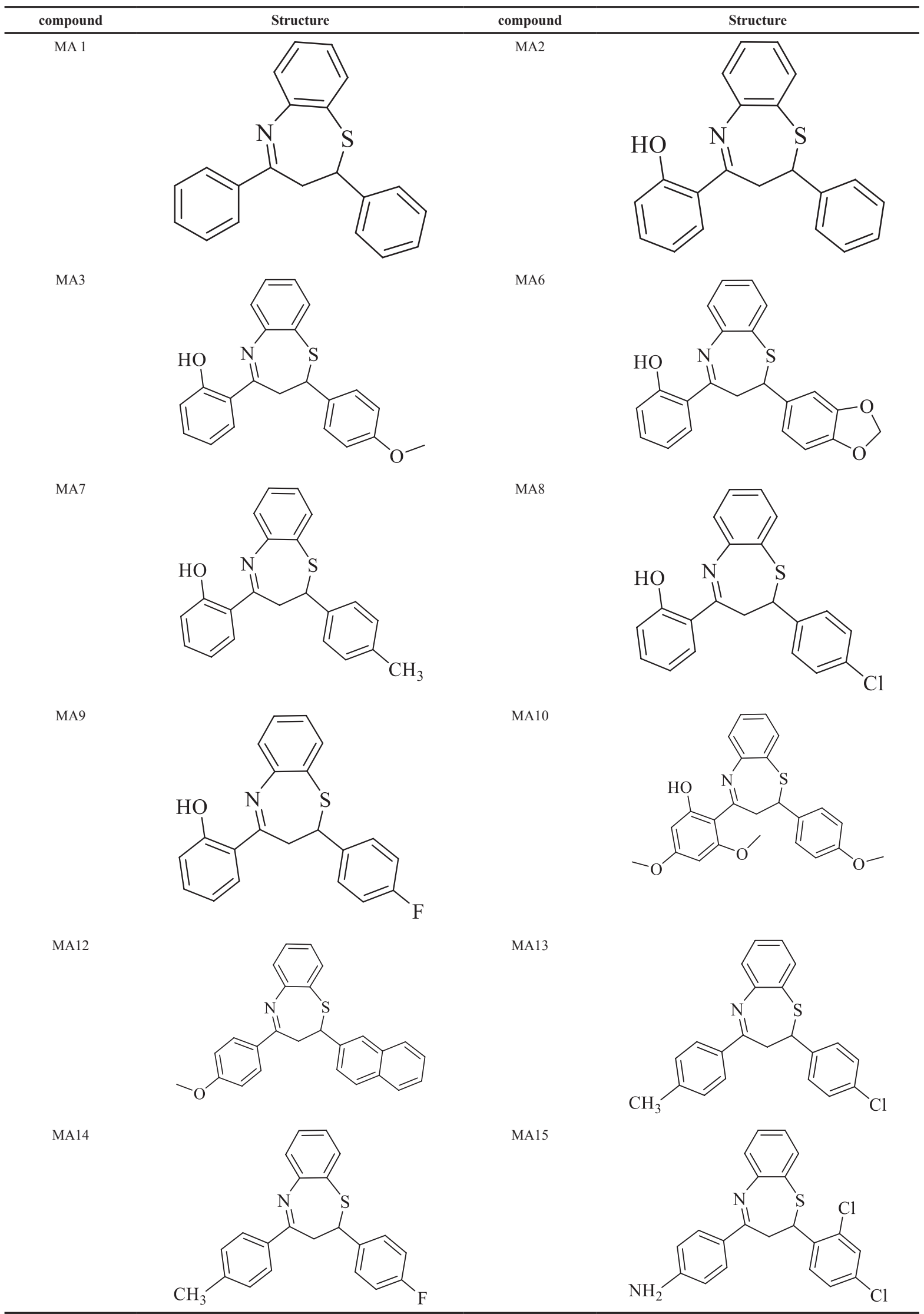




\begin{tabular}{ccc}
\hline compound & compound & Structure \\
\hline DANA & Structure & \\
\hline
\end{tabular}

hour, which was followed by immediate fluorometric measurements of the formed enzyme product: 4-methylumbelliferone using a Modulus Microplate Reader (Turner Biosystem, Sunnyvale CA). The excitation/emission wavelengths were set at $365 / 450 \mathrm{~nm}$. The percentage of neuraminidase inhibition was obtained by fitting the experimental data to the logistic graph.

\section{RESULTS AND DISCUSSION}

\section{Molecular docking}

In this study, we employed molecular docking to study the binding of twelve chalcone-based 1,5-benzothiazepines to neuraminidase from the H1N1 influenza virus (PDB code 3B7E). Specifically, we predicted the bound conformations of the studied compounds inside the active site of neuraminidase (i.e., $\operatorname{Arg} 118$, Arg227, Arg292, Arg371, Arg152, Arg371, Asp151, Arg152, Glu119, Glu277, Asn294, and Leu127). As shown in Table 1, 12 chalcone-based 1,5-benzothiazepine with different functional groups on A and B rings has been synthesized. DANA (Table 1) was used as positive control and docked into the protein active site. Figure 1 shows the spatial arrangement of DANA within the neuraminidase active site. Figure $2 \mathrm{~A}$ shows a three-dimensional arrangement of the lead compound MA10 on the neuraminidase. Figure 2B shows the interactions of specific moieties from MA10 with particular amino acid residues of neuraminidase. We obtained the binding free energy for each compound, which predicts the affinity of the compounds for the enzyme (Table 2). The more negative binding free energy means a stronger binding (Hao, et al, 2013). We have shown free energy of interactions/binding for each compound in comparison to standard neuraminidase inhibitor: DANA (Table 2). Based on these results, DANA with the binding free energy of $-7.1 \mathrm{kcal} / \mathrm{mol}$ can form three hydrogen bonds with amino acid residues Glu128, Val163, and Glu174. Besides, DANA engages in van der Waals interactions with Pro162, Phe173, Lys206, Asn208, and Gly209

The number of hydrogen bonds formed with neuraminidase and the factor of binding (a measure of the probability that a tested compound will bind to the same amino acid residues to which the control compound, DANA binds) calculated by docking may predict, in which compound will be more active neuraminidase inhibitor. Furthermore, root-mean-square deviation (RMSD) is also an important parameter for the prediction of potentially bioactive compounds that ideally should be less than 2 (Yaeghoobi et al., 2016). Usually, the RMSD value is used to validate the docking protocol. The validation of docking protocol means that we need to consider a crystallographic complex protein with ligand in it and perform the docking of the same complex.
As shown in Table 2, the docking results revealed that RMSD for all studied of chalcone-based 1,5-benzothiazepines as well as for DANA is zero. The binding free energy for these benzologs ranged from -8.2 to $-9.3 \mathrm{kcal} / \mathrm{mol}$, which indicated stable interactions with $\mathrm{H} 1 \mathrm{~N} 1$ neuraminidase. The more stable interaction result increased the benzolog's activity (Yaeghoobi et al., 2016). The factor of binding varied among tested benzothiazepines: its values being 1,9 , or 10 . Factor binding is the probability for the occurrence of receptor-ligand binding to the same amino acid residues with the positive control, which is bound/DANA. All benzologs with the factor of binding value 10 except MA7 formed at least one hydrogen bond with amino acid residues inside the catalythic site of the neuraminidase (Table 2). Finally, MA6 engaged in two hydrogen bonds with Val168 and Trp189, which are located at the allosteric sites. We predict MA10 to be the most active compound of all the benzothiazepines despite it formed only one hydrogen bond inside the neuraminidase active site, with Leu127. It is because this residue is situated in the binding site for DANA in the neuraminidase active site (Figure 1), just in the vicinity of Glu128, which forms a hydrogen bond with DANA.

Regarding other types of interactions between MA10 and neuraminidase, we predicted van der Waals interactions with five amino acid residues: Tyr100, Glu174, Trp189, Asn208, and Gly209 (Figure 2). Hydrophobic interactions were also assumed between MA10 ligand and residues Glu128, Val163, and Pro162.

\section{Molecular dynamic}

MD simulations have been widely employed in the current drug design to understand drug-receptor interactions. MD is often used in the first stage of docking, to help predict the binding pose.

MD simulates the dynamic behavior of molecular systems as a function of time; that is, it treats both protein and ligand as flexible entities, computing movements of atoms along time. MD trajectories then can be used as sampling engines; that is, the conformations of proteins created by MD can be employed in molecular docking. This way MD is viewed as a dockingcoupled technique, which is useful not only to assess the stability of docking poses but also to refine/rescore them.

MD simulation can also be used to provide useful information about the completion of the docking prediction (Jasril et al., 2017). Stable energy minimum is the beginning of MD simulation, the protein-ligand system fluctuates around the initial conformation, and the ligand keeps its initial binding mode (Sakano et al., 2016). 


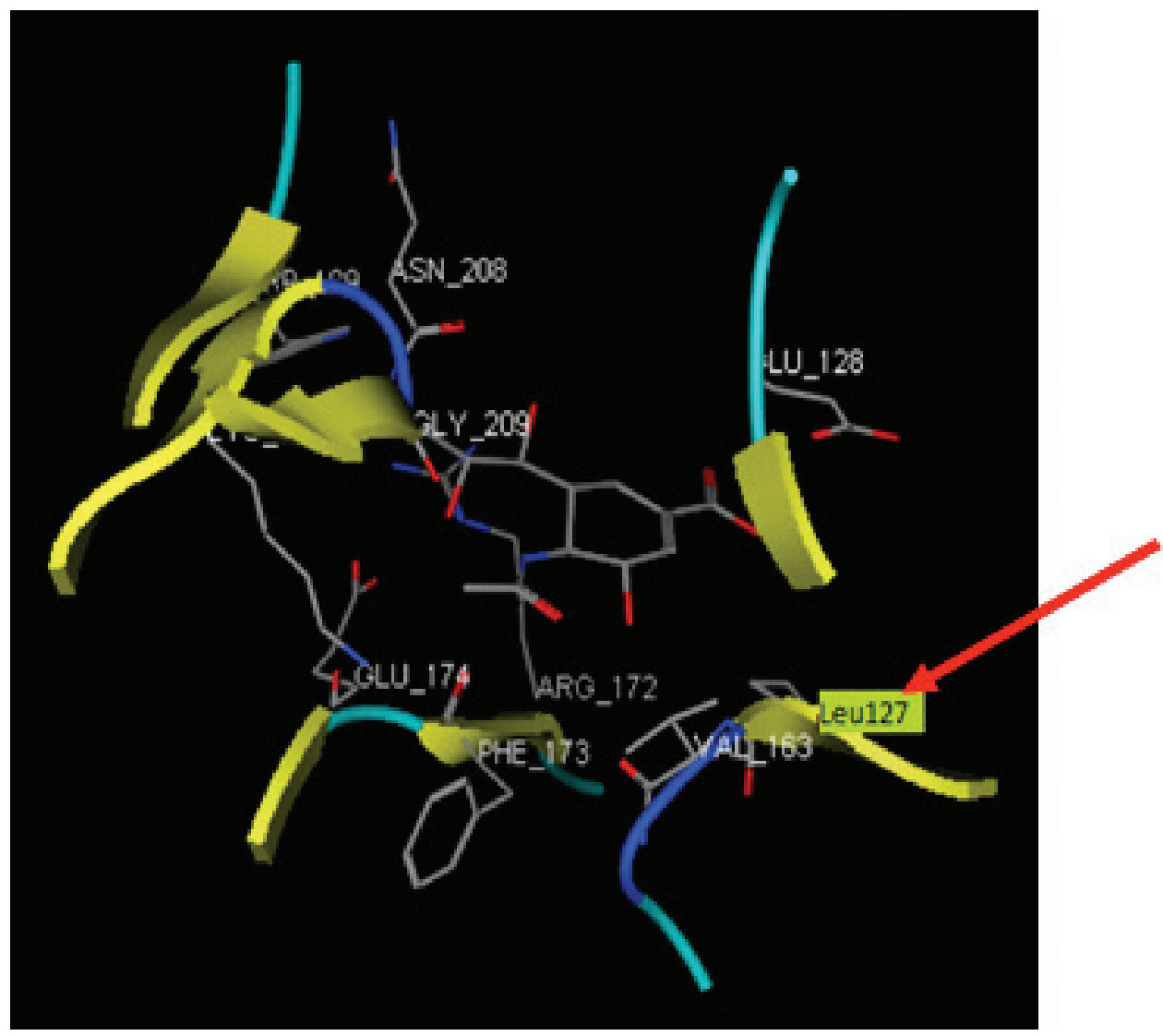

Figure 1. Spatial arrangement of DANA.

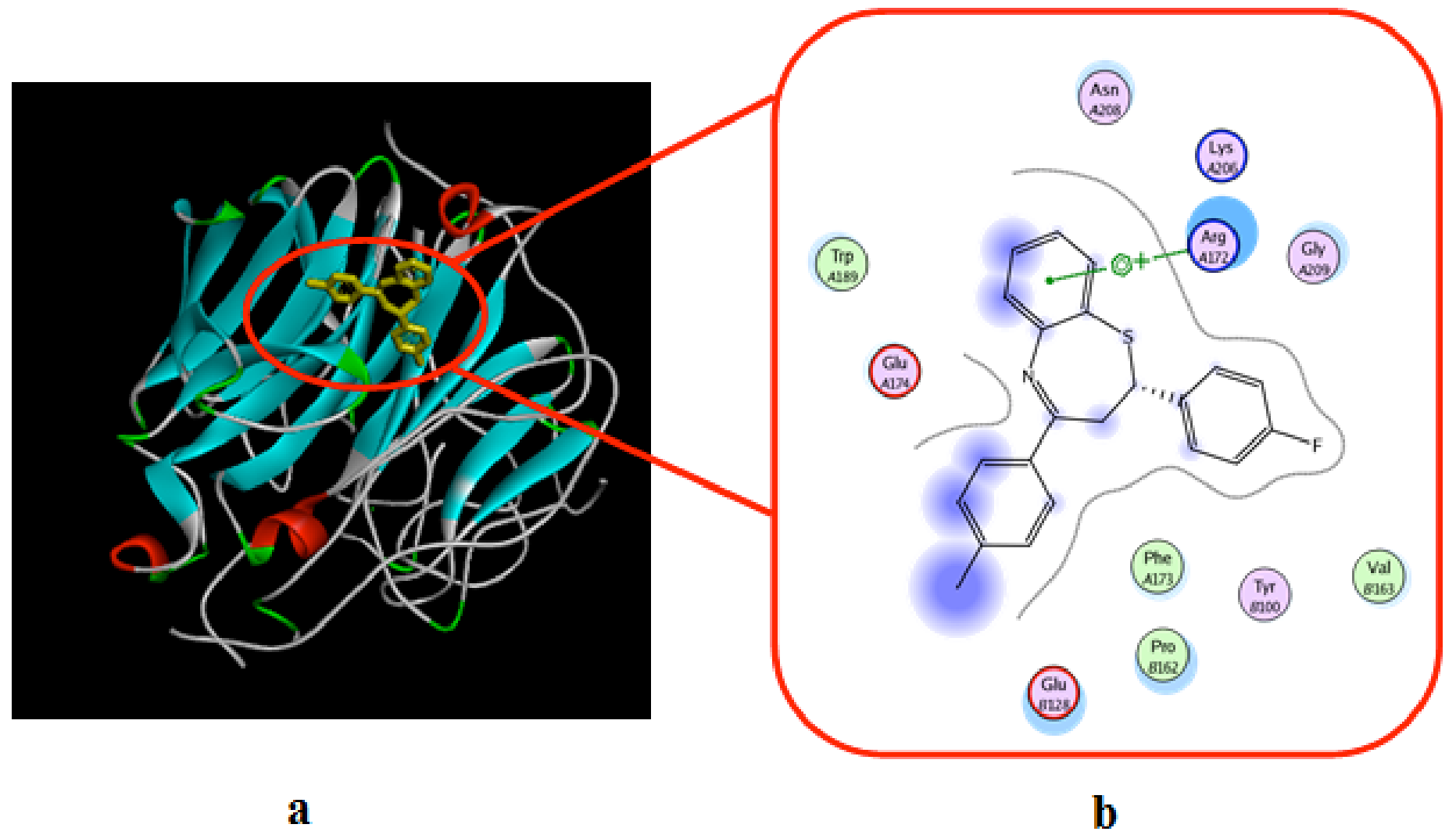

Figure 2. Spatial arrangement of compound MA10. (a) 3D arrangement (b) 2D arrangement. 
In the MD simulations, the affinity of MA10 and DANA for the respective binding sites within $\mathrm{H} 1 \mathrm{~N} 1$ neuraminidase was monitored along $50 \mathrm{~ns}$ at a temperature set to $300 \mathrm{~K}$. The calculated binding energies were -430.5 and $-445.7 \mathrm{kcal} / \mathrm{mol}$ for MA10 and DANA, respectively. The lower the value of the binding energy, the more flexible and the more stable the protein-ligand complex. In other words, the DANA-neuraminidase complex is more stable than the MA10-neuraminidase complex. However, these two values are close enough that we can regard MA10 to be an effective binder of H1N1 neuraminidase. Besides, hydrogen bond distance and free energy of binding over simulation time are important to elucidate the affinity of potential compound The hydrogen bonding distance of DANA with Glu174, Glu128, and Val163 was observed at $2.9 \AA$, and MA10 with Leu127 was

Table 2. Docking results.

\begin{tabular}{cccccc}
\hline \multirow{2}{*}{ Cpd } & \multicolumn{2}{c}{ Parameter } & \multicolumn{2}{c}{ Hydrogen bond } \\
\cline { 2 - 6 } & Binding free energy $(\mathbf{k c a l} / \mathbf{m o l})$ & RMSD & Factor binding & Amino acid & Amount \\
\hline MA 1 & -8.3 & 0.000 & - & - & - \\
MA 2 & -8.4 & 0.000 & 10 & Val168 & 1 \\
MA 3 & -8.4 & 0.000 & 1 & - & - \\
MA 6 & -8.8 & 0.000 & 10 & Trp189, Val168 & 2 \\
MA 7 & -8.5 & 0.000 & 10 & - & - \\
MA 8 & -8.3 & 0.000 & - & - & - \\
MA 9 & -8.6 & 0.000 & 10 & Lys206 & 1 \\
MA 10 & -8.2 & 0.000 & 9 & Leu127 & 1 \\
MA 12 & -9.3 & 0.000 & - & - & - \\
MA 13 & -8.5 & 0.000 & 9 & Lys206 & - \\
MA 14 & -8.8 & 0.000 & 10 & - & 1 \\
MA 15 & -8.5 & 0.000 & - & Glu174, Glu128, Val163 & 3 \\
DANA & -7.1 & 0.000 & & - \\
\hline
\end{tabular}

${ }^{a}$ The probability for the occurrence of receptor-ligand binding to the same amino acid residues with the positive control is bound/DANA.

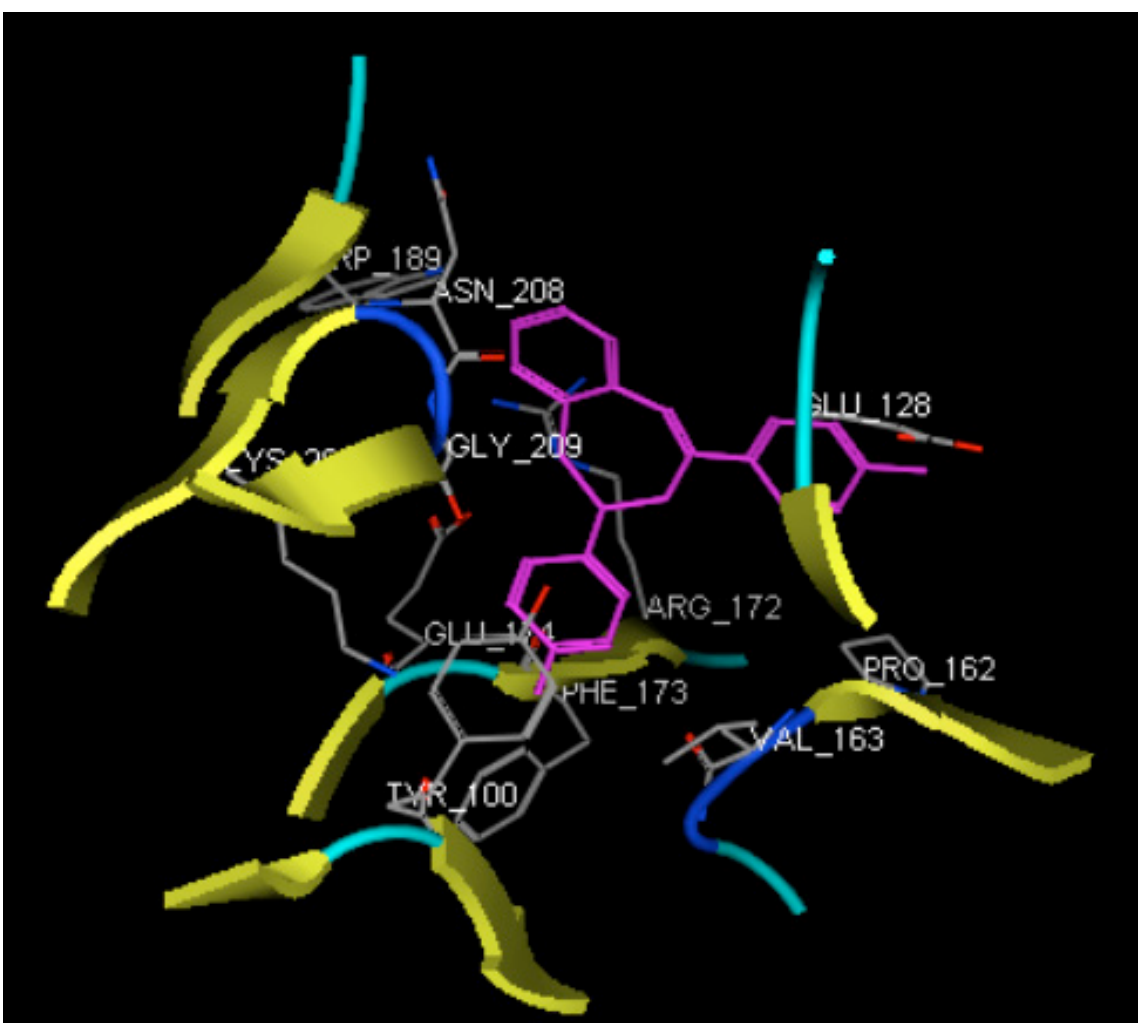

Figure 3. Visualization of binding mode of MA10. 
observed at the distance of $2.9 \AA$. The binding of MA10 inside the binding pocket of neuraminidase is shown in Figure 3.

\section{Pharmacophore generation}

International Union of Pure and Applied Chemistry strictly defines pharmacophore as the ensemble of steric and electronic features that is necessary to ensure the optimal supramolecular interactions with a specific biological target structure and to trigger (or to block) its biological response (Hajare et al., 2011). In this study, we aimed to identify the functionality in chalcone-based 1,5-benzothiazepines, which is the most responsible for the inhibition of H1N1 neuramindase activity after the binding of these benzologs to the enzyme. We have identified pharmocophores in the lead benzolog MA10 and shown in Figure 4: the hydrophobic one (yellow), the hydrogen bond donor (green), and the aromatic ring (pink). We consider these entities of MA10 to be essential for its interactions with $\mathrm{H} 1 \mathrm{~N} 1$ neuraminidase and, hence, for its biological activity.

\section{Absorption, distribution, metabolism, and excretion prediction}

The in silico ADME profile of a drug is also known as its pharmacokinetic profile (Zeynab et. al. 2018). The use of the
ADME profile may reduce the risk of the late-stage attrition of drug development and also for optimizing the screening process. However, it can only be used for the promising or lead compounds (Yamashita and Hashida, 2004).

The Lipinski's rule of five is a rule invented to evaluate the druglikeness of a compound. The rule is employed to determine whether a compound with a certain pharmacological or biological activity has physicochemical properties such that it can be active when administered orally in humans. The majority of orally administered drugs are relatively small and moderately lipophilic molecules. The rule states that an orally active drug may violate zero or maximum one of the following criteria: there can be five or less hydrogen bond donors, there can be ten or less hydrogen bond acceptors, the maximum MW is 500, and the octanol-water partition coefficient $(\log \mathrm{P})$ does not exceed five (Lipinski et al., 2001).

We created ADME profiles of MA10 and DANA, based on in silico calculations. The druglikeness of MA10 was assessed through the Lipinski's rule of five and then compared to that of standard neuraminidase inhibitor: DANA (see Table 4). Based on ADME calculations, MA10 fulfills the Lipinski's rule of five just as DANA does. According to its ADME profile, it appears that MA10 is safe to be used as a potential H1N1 inhibitor.

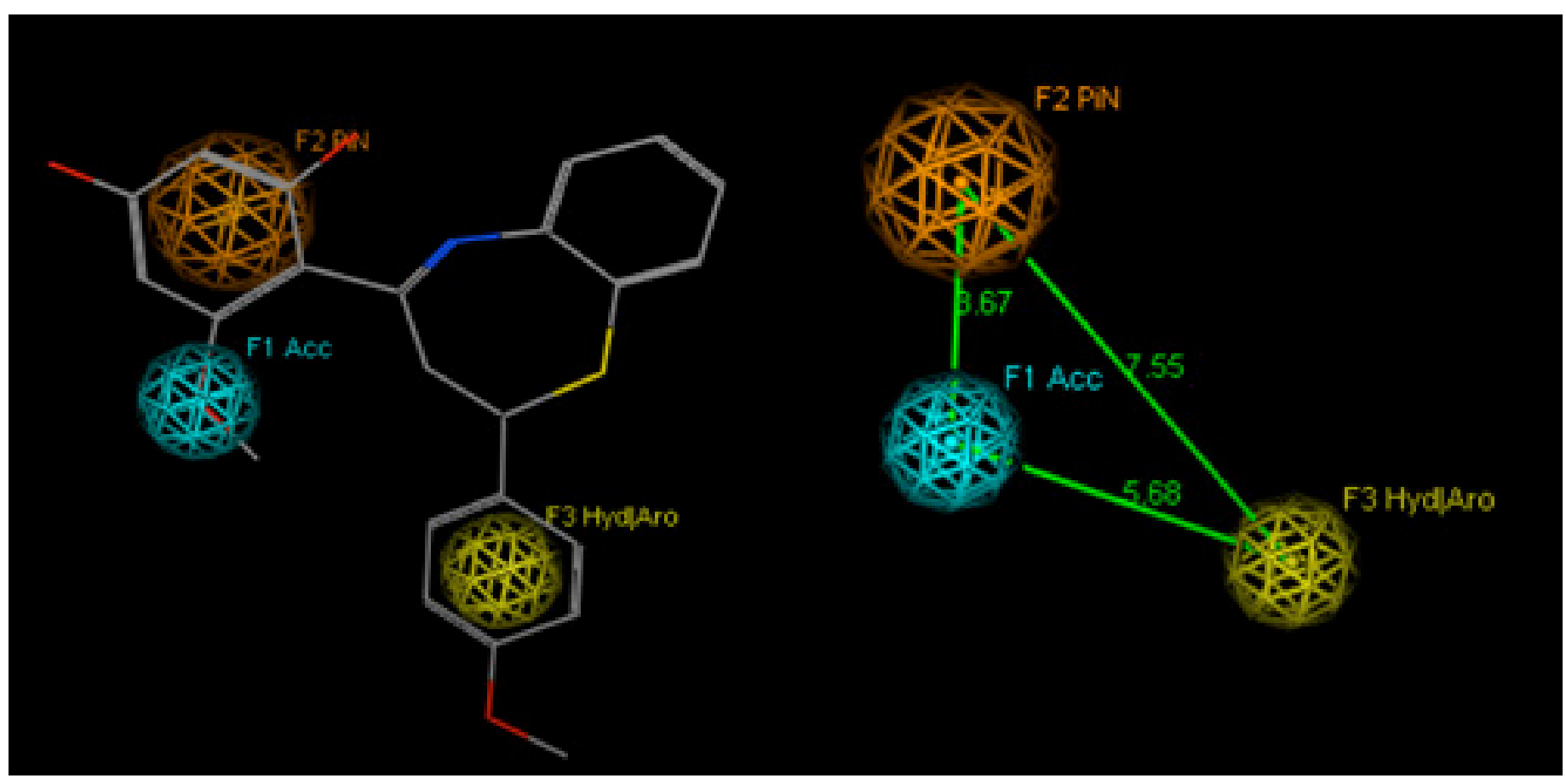

Figure 4. The best pharmacophore hypothesis for compound MA10; distance between pharmacophore features is reported in Angstrom. Pharmacophores are colorcoded with yellow for hydrophobic, blue for hydrogen bond acceptor, and orange for aromatic rings.

Table 3. MD binding free energy conformation.

\begin{tabular}{cccc}
\hline No. & Binding energy $(\mathbf{k c a l} / \mathbf{m o l})$ & Number of hydrogen bonding & Hydrogen bonding distance $(\AA)$ \\
\hline MA10 & -430.5 & Lys127 & 2.9 \\
& & Glu174 & 2.9 \\
\multirow{2}{*}{ DANA } & \multirow{2}{*}{-445.7} & Glu128 & 2.9 \\
& & Val163 & 2.9 \\
\hline
\end{tabular}


Table 4. In silico studies of ADME profiles of compound MA10 and positive control (DANA).

\begin{tabular}{|c|c|c|}
\hline Profiles & MA10 & DANA \\
\hline MW (g/mol) ${ }^{\mathrm{a}}$ & $347.45 \mathrm{~g} / \mathrm{mol}$ & $287.31 \mathrm{~g} / \mathrm{mol}$ \\
\hline Consensus Log $\mathrm{P}_{\mathrm{o} / \mathrm{w}}{ }^{a}$ & 3.57 & 1.01 \\
\hline Hydrogen bond donor $^{\mathrm{a}}$ & 0 & 5 \\
\hline Hydrogen bond acceptor ${ }^{\mathrm{a}}$ & 2 & 6 \\
\hline Rotatable bonds $^{\mathrm{a}}$ & 2 & 6 \\
\hline Druglikeness $^{\mathrm{a}}$ (Lipinski) & Yes & Yes \\
\hline Water solubility $(\mathrm{mg} / \mathrm{ml})^{\mathrm{a}}$ & $4.53 \times 10^{-4}$ (moderately soluble) & $1.51 \times 10^{-1}($ very soluble $)$ \\
\hline CYP1A2 inhibitor ${ }^{\mathrm{a}}$ & Yes & Yes \\
\hline CYP2C19 inhibitor ${ }^{\mathrm{a}}$ & Yes & No \\
\hline CYP2C9 inhibitor ${ }^{\mathrm{a}}$ & Yes & No \\
\hline CYP2D6 inhibitor ${ }^{\mathrm{a}}$ & No & No \\
\hline CYP3A4 inhibitor ${ }^{\mathrm{a}}$ & No & No \\
\hline
\end{tabular}

Calculated using SwisADME server (http://swissadme.ch/index.php).

Log Po/w = lipophilicity; CYP = superfamily of isoenzymes.

Table 5. Percentage of H1N1 neuraminidase inhibitory activity.

\begin{tabular}{ccc}
\hline No. & Compound & Biological activity in $250 \boldsymbol{\mu M}(\%)$ \\
\hline 1 & MA1 & 5.2 \\
2 & MA2 & 5.8 \\
3 & MA3 & 10.5 \\
4 & MA6 & 12.3 \\
5 & MA7 & 6.4 \\
6 & MA8 & 8.5 \\
7 & MA9 & 12.4 \\
8 & MA10 & 25.5 \\
9 & MA12 & 12.7 \\
10 & MA13 & 14.7 \\
11 & MA14 & 15.6 \\
12 & MA15 & 16.8 \\
13 & DANA & 87.5 \\
\hline
\end{tabular}

\section{Biological activity assay}

We have mentioned that chalcone-based 1,5-benzothiazepine derivatives were synthesized by inserting different substituents on 1,5-benzothiazepine core to study the influence of these substituents on H1N1 neuraminidase inhibition. The potency of these compounds to inhibit the neuraminidase in comparison with the standard (and potent) neuraminidase inhibitor, DANA, was evaluated using MUNANA assay.

As shown in Table 5, neither of the tested benzologs has shown a promising inhibition of the neuraminidase. The biological activity ranged from 5.2 (MA1) to $25.5 \%$. (MA10). Although MA10 was predicted to be a promising inhibitor of neuraminidase in the in silico studies and despite the fact that this compound exhibited the best bioactivity among all tested benzologs, the activity of MA10 was too small in comparison to DANA in order for MA10 to become a candidate inhibitor of the neuraminidase. The lower-than-expected inhibitory activity of MA10 is likely due to its low solubility and the lack of flexibility of seven-membered ring of 1,5-benzothiazepine.

\section{CONCLUSION}

The in silico studies revealed that compound MA10 binds favorably to the neuraminidase binding site. Besides, MA10 was able to create few hydrogen bonds with amino acid residues within the active site of the enzyme, and the binding free energy was $-8.8 \mathrm{kcal} / \mathrm{mol}$. We have identified the pharmocophores of MA10 to be hydrophobic, hydrogen bond donor atoms, and aromatic ring, and these entities would be responsible for the biological activity of MA10. MA10 was stable during the molecular dynamic simulation with binding free energy conformation of $-430.5 \mathrm{kcal} /$ mol. It exhibited druglikeness properties based on the Lipinski's rule of five. Moreover, MUNANA assay results showed that compound MA10 was the best inhibitor of neuraminidase of all tested 1,5-benzothiazepines. However, it is not very likely that MA10 will have a future as an antiviral drug against H1N1 due to its relatively low inhibition potency compared to DANA (standard potent neuraminidase inhibitor).

\section{CONFLICTS OF INTEREST}

Authors declared that there are no conflicts of interest.

\section{FUNDING}

We thank STIFAR Riau for the financial support through Hibah STIFAR Riau 2020.

\section{REFERENCES}

Cherkupally SR, Gurrala PR, Adki N, Avula S. Synthesis and biological study of novel methylene-bis-benzofuranyl-[1, 5]-benzothiazepines. Org Commun, 2008; 1(4):84-94.

Chintakrindi AS, Gohil DJ, Kothari ST, Chowdhary AS, Kanyalkar MA. Design, synthesis and evaluation of chalcones as H1N1Neuraminidase inhibitors. Med Chem Res, 2018; 27:1013-25.

Dandia A, Upreti M, Rani B, Pant UC, Gupta IJ. Synthesis and antimicrobial evaluation ofsome new fluorinated spiro[1,5]-benzothiazepin2,3'[3'H]-indol]-2'(1'H)-ones. J Fluor Chem, 1998; 91:171-4.

Fernández F, Ruiz P. Psychiatric aspects of HIV/AIDS. Lippincott William \& Wilkins, Philadelphia, PA, 2006.

Geyer HM, Watzman N, Buckley JP. Effects of a tranquilizer and two antidepressants on learned and unlearned behaviors. J Pharmacol Sci. 1970; 59:964-8.

Hajare RA, Landge ST, Darvhekar DM, Chandewar AV. Pharmacophore in drug design and discovery. Pharmacophore in drug design and discovery. The pharma Review (January-February), Kongpost publication, pp 45-149, 2011.

Hao Z, Wei H, Gu He, Cheng P, Fengbo W, Liang O. Molecular dynamics simulation of tryptophan hydroxylase-1: binding modes and free energy analysis to phenylalanine derivative inhibitors. Int J Mol Sci, 2013; 14:9947-62.

Jasril J, Ikhtiarudin I, Zamri A, Teruna HY, Frimayanti N. New fluorinated chalcone and pyrazoline analogs: Synthesis, docking and molecular dynamic studies as anticancer agents. Thai J Pharm Sci, 2017; 41:93-8.

Kawashima. Co. Lt. Benzothiazepine derivative. Jap. Pat. 60056972. Chem Abstr, 1985; 103:105014.

Kugita H, Inoue H, Ikezaki M, Konda M, Takeo S. Synthesis of 1, 5-benzothiazepine derivatives.III. Chem Pharm Bull, 1971; 19:595-602.

Leotis LB, Cummings RD, Steinhauer DA. The interplay between the host receptor and influenza virus hemagglutinin and neuraminidase. Int J Mol Sci, 2017; 18(7):1541-63.

Lipinski CA, Lombardo F, Dominy BW, Feeney PJ. Experimental and computational approaches to estimate solubility and permeablility in drug:discovery and development setting. Adv Drug Deliv Rev, 2001; 46(1$3): 3-26$. 
Maksum RM. Imunologi dan virologi edisi pertama. Penerbit PT. ISFI, Jakarta Barat, Indonesia, pp 164-8, 2010.

Neni F, Ihsan I, Rahma D, Tiara TA, Fri M, Adel ZA. Computational approach to drug discovery: search for chalcone analogues as the potential candidates for anti colorectal cancer (HT29). Walailak J Sci Technol, 2020; 12(2):64-74.

Page C, Michael C, Sutter M, Walker M, Hoffman B. Integrated pharmacology. Elsevier, New York, NY, 2002.

Peter KCC, Tommy WCL, Eric CMH, Peter CKL, Anita YYN, Mary YYL, Wilina WLL. Oseltamivir and amantadine resistant a (H1N1). Emerg Infect Dis, 2009; 15:966-8.

Reddy JR, Ashok D, Sharma PN. Synthesis of 4,6-bis(2'substituted-2',3'-dihydro-1,5-benzothiazepin-4'-yl) resorcinols as potential antifeedants. Indian J Chem B, 1993; 32:404-6.

Sakano T, Mahamood MI, Yamashita T, Fujitani H. Molecular dynamics analysis to evaluate docking pose prediction. Biophys Physicobiol, 2016; 13:181-94.

Taubenberger JK, Morens DM. Influenza: the mother of all pandemic. Emerg Infect Dis, 2006; 12:15-22.

Wermuth CG. Glossary of term used in medicinal chemistry. Pure Appl Chem, 1998; 70:1129-43.

Yaeghoobi M. Synthesis of chalcone based-six and seven membered heterocyclic compounds and their biological activities against H1N1 virus [thesis]. University of Malaya, Kuala Lumpur, Malaysia, pp 121-33, 2012.
Yaeghoobi M, Neni F, Chin FC, Kusaira KI, Belal ON, Sharifuddin MZ, Zanariah A, Habibah AW, Noorsaadah AR. QSAR, in silico docking and in vitro evaluation of chalcone derivatives as potential inhibitors for H1N1 virus neuraminidase. Med Chem Res, 2016; 25:213342.

Yamashita F, Hashida M. Drug metab. Pharmacokinet, 2004; 19(5):327-38.

Yu K, Luo C, Qin G, Xu Z, Li N, Liu H, Shen X, Ma J, Wang $\mathrm{Q}$, Yang $\mathrm{C}$, Zhu $\mathrm{W}$, Jiang $\mathrm{H}$. Why are oseltamivir and zanamivir effective against the newly emerged influenza a virus (A/H1N1)? Cell Res, 2009; 19:1221-4.

Zeynab AL, Mahdieh S, Zahra H, Sepehr S, Rezvan Z, Mohammad RA, Eskandanr A, Afshin Z. Design, synthesis, molecular modeling, in silico ADME studies and anti- HIV-1 assay of new diazocoumarin derivatives. Iran J Pharm Res, 2018; 17(2):65-77.

How to cite this article:

Frimayanti N, Yaeghoobi M, Namavar H, Ikhtiarudin I, Afzali M. In silico studies and biological evaluation of chalcone-based 1,5-benzothiazepines as new potential H1N1 neuraminidase inhibitors. J Appl Pharm Sci, 2020; 10(10):086-094. 